\title{
Las antropologías de los antropólogos más allá de la academia
}

\author{
Anthropologies of anthropologists beyond university
}

José Juncosa *

Naturaleza y cultura en América Latina. Escenarios para un modelo de desarrollo no civilizatorio (Memorias del XVIII Foro de Estudiantes Latinoamericanos de Antropología y Arqueología-FELAA. Quito, 17 al 23 de julio del 2011). Eloy Alfaro Reyes, Katterine Enríquez y Yolanda Flores (compiladores). Ediciones Abya-Yala/UPS. Quito, 2012.

El propósito de esta reseña es identificar en la obra analizada, compilada y editada por estudiantes de la Carrera de Antropología Aplicada de la Universidad Politécnica Salesiana,las expectativas que los estudiantes latinoamericanos le atribuyen a la antropología en referencia a los usos sociales y apuestas existenciales que despliegan a través de ella y más allá de ella. El Foro de Estudiantes Latinoamericanos de Antropología y Arqueología (FELAA) nació en 1994 y tuvo lugar por primera vez en Costa Rica. Desde entonces, se reedita anualmente de manera ininterrumpida y es totalmente gestionado por los mismos estudiantes. Que nosotros sepamos, es el único caso de articulación permanente y autónoma de estudiantes universitarios en América Latina y constituye de por sí un hecho social del campo académico que es necesario comprender y digno de una investigación de mayor profundidad.

La versión del XVIII Foro se realizó en Quito (17 al 23 de julio del 2011), fue acogido por la Carrera de Antropología de la Universidad Politécnica Salesiana y se desarrolló mediante la modalidad de campamento académico en la sede El Girón de la misma universidad. Algunas cifras nos dan la idea del tipo de participación y alcance de este Foro (Alfaro et al., 2012: 15 ss.) al que asistieron 233 estudiantes provenientes de Chile (37), Colombia (105), México (14), Perú (4), Brasil (2), Bolivia (1), Uruguay (10), España (1) y Ecuador (59).

Antropólogo , Director de la Carrera de Antropología Aplicada de la Universidad Politécnica Salesiana del Ecuador. 
El 60\% de los asistentes fueron mujeres y se desplegaron 75 participaciones de diverso tipo.

La heterogeneidad de los participantes se revela, además, en los siguientes elementos: la mayoría proviene de universidades públicas, pero también de privadas; desarrollan su formación mediante modalidades diversas (presencial y a distancia) y supone la interacción de estudiantes adultos (particularmente aquellos que pertenecen a la modalidad a distancia de la Carrera de Antropología Aplicada de la UPS) con estudiantes más jóvenes; por último, el foro convocó a estudiantes de comunicación, sociología y pedagogía, demostrando que la antropología es un ámbito idóneo para el debate de temas más amplios y transversales y, finalmente, el foro fue el escenario para el encuentro de estudiantes provenientes de organizaciones indígenas, como las del Cabildo de Jóvenes Indígenas del Cauca, y afroamericanas.

El foro enfatizó los siguientes aspectos, identificados a partir del diálogo con el equipo organizador y las observaciones personales:

1. Integración de diversas modalidades comunicativas tradicionales (conferencias magistrales, simposios, ponencias) con otras de carácter participativo y colaborativo (talleres, muestras de cine y fotografía, asambleas, encuentros, etc.);

2. Realización de trabajo de campo por equipos en cuatro puntos del país: Lago Agrio (Amazonía), Muisne (Costa), Quimsacocha (Cuenca) y Tulipe (Noroccidente de Quito) para, a través del contacto con la comunidad, acceder a las posiciones sobre la explotación petrolera, la degradación del manglar, la minería y el turismo comunitario;

3. Cuidado explícito y manifiesto por la calidad académica de las intervenciones. Respecto a este último rasgo, destacamos que cada una de las ponencias estudiantiles publicadas (31 en total) fue objeto de los estándares de arbitraje académico que incluye el veredicto de los docentes de las respectivas universidades.

La documentación de las memorias del FELAA de Quito se realizó a través de dos publicaciones y un video. El primer producto editorial recoge las memorias del foro previo, realizado en abril del 2011 en la Universidad Politécnica Salesiana (La arqueología y la antropología en Ecuador. Escenarios, 
retos y perspectivas. Abya-Yala/UPS, Quito, 2011, 180 páginas). Se trata de una suerte de diagnóstico sobre la situación de la arqueología (ver los artículos de Delgado y Rivadeneira) y de la antropología ecuatorianas mediante el análisis de los retos actuales según los siguientes énfasis: los desafíos de la profesión en la academia (ver los artículos de Alfaro Reyes, Eguiguren y Sanchez-Parga); en la transformación social (ver los artículos de Guerrero, Herrera, Viteri y Chalá); y en los espacios del Estado y las políticas públicas (ver el artículo de Larrea y el de Delgado, para la arqueología). El segundo consiste de las memorias completas del Foro, un extenso libro de 604 páginas que será objeto de la presente recensión. Finalmente, el Foro produjo un video de 30 minutos de duración que recoge la documentación gráfica de los testimonios, actividades, resultados y actores.

Lo primero que destacamos de estas memorias es el descentramiento disciplinar o la indisciplina que supone articular el foro, según lo expresa el índice del tomo, sin casi tomar en cuenta el itinerario temático regido por el clásico elenco de disciplinas y subdisciplinas de la antropología y la arqueología para, en cambio, organizar el debate de acuerdo a los campos de la vida sociocultural a los que se supone que las disciplinas deben contribuir. Esta es una de las diferencias respecto a las memorias de foros anteriores, No se trata, por tanto, de una innovación menor sino de la puesta en práctica de un saludable enfoque epistémico que ubica el punto de tensión por fuera del marco disciplinar y coloca el criterio de comparecencia del conocimiento fuera de sí mismo, en la sociedad.

Las ponencias, en efecto, se ordenan según los siguientes temas: 1. Antropología e interculturalidad, 2. Antropología política, 3. Antropología urbana, 4. Antropología visual, 5. Antropología y educación, 6. Cuerpo, género y antropología, 7. Cultura y naturaleza, 8. Estética y antropología, 9. Patrimonio, 10. Salud y antropología. De hecho, muy pocas ponencias se basan en la antropología o la arqueología como objetos de conocimiento disciplinarios, pero siempre desde una perspectiva crítica respecto a las demandas y emergencias sociales. Algunas corresponden a profesores invitados, como Natalia Sotomayor, de Ecuador (La perspectiva de género en la Antropología); y Miguel Eduardo Cárdenas, de Colombia (Retos y desafios para la Antropología en América Latina). Otras que forman parte de este grupo fueron elaboradas por estudiantes, como por ejemplo, Diego F. Porras, de México (Antropología política-econó- 
mica como recurso para estudios del narcotráfico); y Jeff Contreras y Máximo Arteaga (Una aproximación a la arqueología por contrato en el Perú). Destaco en este punto que la experiencia sentida (el saber sentido de Benjamin) de la crisis civilizatoria y la certeza de que "muchas cosas andan mal" en el mundo y no sólo en la academia (Cárdenas Rivera: 2012: 44 ss.) el mejor lugar desde el cual identificar los retos de la antropología.

Lo segundo a destacar es que el Foro responde, en gran medida, a una de las tendencias identificadas por el Grupo de Trabajo sobre Universidades Latinoamericanas de la CLACSO según el cual, y en el contexto del proceso político latinoamericano y de las protestas juveniles, las universidades, sobre todo las públicas, "han sido desafiadas a asumir un papel estratégico en la búsqueda de alternativas al neoliberalismo" (Leher 2010: 9) de tal manera que no pocas contribuciones son contestatarias a los modelos de sociedad y de educación superior basada en la comodificación de las universidades (ídem: 11) y expresan orientaciones mucho más críticas y contestatarias. Esta tendencia se expresa, por ejemplo, en la selección de conocidos ponentes/activistas invitados y líderes indígenas opositores al modelo de desarrollo extractivista de Ecuador, como Édgar Isch, Pablo Fajardo y Humberto Cholango. De los estudiantes, algunas de las ponencias que reflejan la crítica a este modelo de desarrollo y a sus orientaciones civilizatorias consumistas y a contravía de los derechos de la naturaleza son la de Soledad Vogliano y María Fernanda Vallejo ("Insurgir en el ombligo de la bestia". Re-existencia y descolonialidad en el seno del desarrollo), para el caso de Ecuador; la de Laura Vanegas y Luis Plazas (Amazonía: la disputa entre los espacios ancestrales y los intereses imperialistas), para el caso colombiano; y la de Fabiola Albornoz (¿Educar para colonizar o descolonizar para educar?), para el caso chileno. Esta última se inscribe en el movimiento estudiantil basado en la conexión entre la economía política y los sistemas educativos.

El tercer aspecto a relievar es la presencia de búsquedas, algunas de ellas alimentadas por la reflexión en torno al género y al movimiento feminista, que reivindican estilos de vida y prácticas individuales basadas en el reconocimiento de la corporalidad negada y de nuevas sexualidades estableciendo una crítica a los patrones culturales de convivencia basados en el machismo y la homofobia, en la sexualidad que encierra el erotismo en la reproducción y subordinan el mundo de las emociones a la racionalidad intelectual. Además de la crítica 
cultural que estos artículos plantean, es necesario reconocer también que se trata de la emergencia de nuevas maneras de construir subjetividades a partir del reconocimiento del propio cuerpo - individual y social - como lenguaje y territorio. Asimismo, me llama muchísimo la atención que en la crítica y la construcción de subjetividades predomina el recurso a la danza, la sensibilidad estética y la reafirmación de la sensualidad, en tanto lugares antropológicos de modelación identitaria, actuación social y conocimiento.

El testimonio de la antropóloga Natalia Sotomayor se hace eco de esta veta y la lee desde los posibles desafíos que supone para la antropología:

...quiero repetir que es nuestra responsabilidad observar cómo los sujetos concretos están reorganizando sus vidas en torno a los cambios que ocurren a nivel mundial. Esto implica admitir la posibilidad de cambios en la vida humana a todo nivel, desde los símbolos de género, hasta manifestaciones concretas en otros ámbitos de la vida humana.

Implica reconocer, por ejemplo, que los movimientos solidarios como el Sumak Kawsay, la democracia de la tierra y otras propuestas alternativas al sistema mundial dominante no son solamente un concepto sino que están conformados por prácticas múltiples y diversas de personas que reivindican sus bienes y espacios comunales, sus recursos, sus medios de vida, sus libertades, su dignidad, sus identidades y su paz. Son movimientos polifacéticos y múltiples que están transformando al sistema mundial. (Sotomayor 2012, 106)

Me animo a pensar que el congreso, en general, supone una crítica velada no solo a las formas tradicionales de activismo contundente de transformación social que no siempre modelan subjetividades alternativas y alterativas sino también a las formas de aprendizaje -tradicionales o contemporáneas- que mutilan las conexiones no solo entre las disciplinas sino también respecto a las emociones y los sentidos; pero más allá de la crítica predomina la propuesta y la reivindicación del cuerpo y la sensibilidad.

Algunas de las ponencias ilustrativas de esta tendencia cultural son las que forman parte del grupo Cuerpo, género y antropología elaboradas por Ximena Batista, de México; Guadalupe Rivadeneira, de Ecuador; y por el grupo conformado por María V. Durán, Natalia Acuña y Natalia Toro, de Colombia. Los títulos de las ponencias cobijadas bajo el descriptor Estética y Antropología 
son muy relevadores: Antropología y danza (de Ada Rabago, México); "Cuerpo como territorio". Intercambio artístico/educativo. Reseña de viaje (María Elena Retamal R., Chile); Cuerpo develado como sujeto cognoscente en el arte (Yolanda Flores, Ecuador); Prácticas performáticas chilenas. Ejercicios de evidencias y reivindicaciones de la memoria corporal en jóvenes artistas contemporáneos (Paula Miranda Vera, Chile).

Me absuelvo de considerar aquí otras contribuciones mejor relacionadas con lo que el sentido común circulante considera el objeto clásico e inamovible de la antropología: los pueblos indígenas y sus expresiones identitarias y simbólicas, las que si bien ocupan un lugar importante no desplazan el eje de discusión que se constituye alrededor de los énfasis arriba señalados. Parecería, entonces, que la antropología, cada vez más, se sacude del encargo (... de la carga) que le atribuye la misión de construir clasificaciones sociales, sobrevisibilizar las diferencias para, en última instancia, sobredeterminarlas política y económicamente.

Pensamos que una obra, de por sí extensa y compuesta de aportes heterogéneos, merece lecturas más atentas y complejas sin olvidar que se ha constituido en la memoria de un espacio de encuentro de actores vinculados con las nuevas emergencias sociales y sus demandas que atraviesan la academia al mismo tiempo que la supera, configurando biografías - constituidas desde las aulas pero también constituyentes de la academia -proyectadas más allá de las incidencias circunscritas a la formación profesional.

Como ya se vuelve habitual en las conversaciones entre colegas, este tomo alimenta el reconocimiento creciente de que, afortunadamente, lo más interesante de la antropología no ocurre (solo) al interno de la disciplina sino fuera de ella. La lectura de estas memorias es no solo recomendable sino también necesaria para los docentes de esta y otras disciplinas, pues ofrece muchos elementos que permitirán calibrar y reorientar el aprendizaje a partir de las expectativas, posiciones y reafirmaciones existenciales que los estudiantes vuelcan en el aprendizaje de la antropología. 


\section{Bibliografía}

ALBORNOZ TAPIA, Fabiola

2012 “Educar para colonizar o descolonizar para educar?”, en: Eloy Alfaro R., Katterine Enríquez y Yolanda Flores (comp.). Naturaleza y Cultura en América Latina. Escenarios para un modelo de desarrollo no civilizatorio. (Memorias del XVIII Foro de Estudiantes Latinoamericanos de Antropología y Arqueología - FELAA. Quito, 17-23 de julio del 2011). Quito: Ediciones Abya-Yala/UPS.

ALFARO REYES, Eloy

2011 "Lectura latinoamericana de las tesis sobre las historias de Walter Benjamin: desafíos para la Antropología", en: Katterine Enríquez (compilación y edición). La arqueología y la antropología en Ecuador. Escenarios, retos y perspectivas. Quito: Ediciones Abya-Yala/UPS.

ALFARO REYES, Eloy, Katterine Enríquez y Yolanda Flores (eds.)

2012 Naturaleza y Cultura en América Latina. Escenarios para un modelo de desarrollo no civilizatorio. (Memorias del XVIII Foro de Estudiantes Latinoamericanos de Antropología y Arqueología - FELAA. Quito, 17-23 de julio del 2011). Quito: Ediciones Abya-Yala/UPS.

BATISTA ORDAZ, Ximena

2012 "Dos cuerpos iguales en la misma cama. Hacia una legitimación de prácticas sexuales no reproductivas desde la diversidad sexual humana", en: Eloy Alfaro R., Katterine Enríquez y Yolanda Flores (comp.). Naturaleza y Cultura en América Latina. Escenarios para un modelo de desarrollo no civilizatorio. (Memorias del XVIII Foro de Estudiantes Latinoamericanos de Antropología y Arqueología - FELAA. Quito, 17-23 de julio del 2011). Quito: Ediciones Abya-Yala/UPS.

CÁRDENAS RIVERA, Miguel

2012 "Retos y desafíos para la Antropología en América Latina", en: Eloy Alfaro R., Katterine Enríquez y Yolanda Flores (comp.). Naturaleza y Cultura en América Latina. Escenarios para un modelo de desarrollo no civilizatorio. (Memorias del XVIII Foro de Estudiantes Latinoamericanos de Antropología y Arqueología - FELAA. Quito, 17-23 de julio del 2011). Quito: Ediciones Abya-Yala/UPS.

CHALÁ CRUZ, José

2011 "La antropología como compromiso", en: Katterine Enríquez (compilación y edición). La arqueología y la antropología en Ecuador. Escenarios, retos y perspectivas. Quito: Ediciones Abya-Yala/UPS. 
CHOLANGO, Humberto

2012 "Los Pueblos y nacionalidades del Ecuador frente al estractivismo", en: Eloy Alfaro R., Katterine Enríquez y Yolanda Flores (comp.). Naturaleza y Cultura en América Latina. Escenarios para un modelo de desarrollo no civilizatorio. (Memorias del XVIII Foro de Estudiantes Latinoamericanos de Antropología y Arqueología - FELAA. Quito, 17-23 de julio del 2011). Quito: Ediciones Abya-Yala/UPS.

CONTRERAS SOTO, Jeff y Máximo Arteaga Mendoza

2012 "Una aproximación a la arqueología por contrato en el Perú", en: Eloy Alfaro R., Katterine Enríquez y Yolanda Flores (comp.). Naturaleza y Cultura en América Latina. Escenarios para un modelo de desarrollo no civilizatorio. (Memorias del XVIII Foro de Estudiantes Latinoamericanos de Antropología y Arqueología - FELAA. Quito, 17-23 de julio del 2011). Quito: Ediciones Abya-Yala/UPS.

DELGADO ESPINOZA, Florencio

2011). "La Arqueología ecuatoriana en el siglo XXI: entre la academia y la Arqueología Aplicada", en:KatterineEnríquez (compilación y edición). La arqueología y la antropología en Ecuador. Escenarios, retos y perspectivas. Quito: Ediciones Abya-Yala/UPS.

DURÁN CELEMIN, María, Natalia Acuña P. y Natalia Toro R.

2012 "Movilizándonos en las tramas del género: una geografía feminista del transporte público masivo de Bogotá”, en: Eloy Alfaro R., Katterine Enríquez y Yolanda Flores (comp.). Naturaleza y Cultura en América Latina. Escenarios para un modelo de desarrollo no civilizatorio. (Memorias del XVIII Foro de Estudiantes Latinoamericanos de Antropología y Arqueología - FELAA. Quito, 17-23 de julio del 2011). Quito: Ediciones Abya-Yala/UPS.

EGUIGUREN EGUIGUREN, Amparo

2011 "La esperanza es lo último que se pierde... Prácticas profesionales antropológicas del Ecuador", en: Katterine Enríquez (compilación y edición). La arqueología y la antropología en Ecuador. Escenarios, retos y perspectivas. Quito: Ediciones Abya-Yala/UPS.

ENRÍQUEZ, Katterine (compilación y edición)

2011 La arqueología y la antropología en Ecuador. Escenarios, retos y perspectivas. Quito: Ediciones Abya-Yala/UPS.

ENRÍQUEZ, Katterine

2012 "Informe. Foro de Estudiantes Latinoamericanos de Arqueología y Antropología", en: Eloy Alfaro Reyes, Katterine Enríquez y Yolanda Flores (eds.). Naturaleza y Cultura en América Latina. Escenarios para un modelo de desarrollo no civilizatorio. (Memorias del XVIII Foro de Estudiantes Latinoamericanos de Antropología y Arqueología - FELAA. Quito, 17-23 de julio del 2011). Quito: Ediciones Abya-Yala/UPS. 
FAJARDO, Pablo

2012 "Frente de Defensa de la Amazonía. El juicio a la petrolera Chevron-Texaco", en: Eloy Alfaro R., Katterine Enríquez y Yolanda Flores (comp.). Naturaleza y Cultura en América Latina. Escenarios para un modelo de desarrollo no civilizatorio. (Memorias del XVIII Foro de Estudiantes Latinoamericanos de Antropología y Arqueología - FELAA. Quito, 17-23 de julio del 2011). Quito: Ediciones Abya-Yala/UPS.

FLORES, Yolanda

2012 "Cuerpo develado como sujeto cognoscente en el arte", en: Eloy Alfaro R., Katterine Enríquez y Yolanda Flores (comp.). Naturaleza y Cultura en América Latina. Escenarios para un modelo de desarrollo no civilizatorio. (Memorias del XVIII Foro de Estudiantes Latinoamericanos de Antropología y Arqueología - FELAA. Quito, 17-23 de julio del 2011). Quito: Ediciones Abya-Yala/UPS.

GUERRERO ARIAS, Patricio

2011 "Por una antropología del corazonar comprometida con la vida", en: Katterine Enríquez (compilación y edición). La arqueología y la antropología en Ecuador. Escenarios, retos y perspectivas. Quito: Ediciones Abya-Yala/ UPS.

HERRERA MONTERO, Luis

2011 "La antropología aplicada como parte de los procesos de transformación social", en: Katterine Enríquez (compilación y edición). La arqueología y la antropología en Ecuador. Escenarios, retos y perspectivas. Quito: Ediciones

ISCH, Édgar Abya-Yala/UPS.

2012 “Antropología, derechos y naturaleza: visiones no civilizatorias”, en: Eloy Alfaro R., Katterine Enríquez y Yolanda Flores (comp.). Naturaleza y Cultura en América Latina. Escenarios para un modelo de desarrollo no civilizatorio. (Memorias del XVIII Foro de Estudiantes Latinoamericanos de Antropología y Arqueología - FELAA. Quito, 17-23 de julio del 2011). Quito: Ediciones Abya-Yala/UPS.

\section{LARREA, Ana María}

2011 "La antropología en los escenarios de trabajo en el Estado", en: Katterine Enríquez (compilación y edición). La arqueología y la antropología en Ecuador. Escenarios, retos y perspectivas. Quito: Ediciones Abya-Yala/ UPS.

LEHER, Roberto (compilador)

2010 Por una reforma radical de las universidades latinoamericanas. Rosario: Clacso y Homo Sapiens. 
MIRANDA VELA, Paula

2012 "Prácticas performáticas chilenas. Ejercicios de evidencias y reivindicaciones de la memoria corporal en jóvenes artistas contemporáneos", en: Eloy Alfaro R., Katterine Enríquez y Yolanda Flores (comp.). Naturaleza y Cultura en América Latina. Escenarios para un modelo de desarrollo no civilizatorio. (Memorias del XVIII Foro de Estudiantes Latinoamericanos de Antropología y Arqueología - FELAA. Quito, 17-23 de julio del 2011). Quito: Ediciones Abya-Yala/UPS.

PORRAS MARULANDA, Diego

2012 "Antropología política-económica como recurso para estudios del narcotráfico", en: Eloy Alfaro R., Katterine Enríquez y Yolanda Flores (comp.). Naturaleza y Cultura en América Latina. Escenarios para un modelo de desarrollo no civilizatorio. (Memorias del XVIII Foro de Estudiantes Latinoamericanos de Antropología y Arqueología - FELAA. Quito, 17-23 de julio del 2011). Quito: Ediciones Abya-Yala/UPS.

RABAGO, Ada

2012 “Antropología y danza”, en: Eloy Alfaro R., Katterine Enríquez y Yolanda Flores (comp.). Naturaleza y Cultura en América Latina. Escenarios para un modelo de desarrollo no civilizatorio. (Memorias del XVIII Foro de Estudiantes Latinoamericanos de Antropología y Arqueología - FELAA. Quito, 17-23 de julio del 2011). Quito: Ediciones Abya-Yala/UPS.

RETAMAL, María R.

2012). “Cuerpo como territorio'. Intercambio artístico/educativo. Reseña de viaje”, en: Eloy Alfaro R., Katterine Enríquez y Yolanda Flores (comp.). Naturaleza y Cultura en América Latina. Escenarios para un modelo de desarrollo no civilizatorio. (Memorias del XVIII Foro de Estudiantes Latinoamericanos de Antropología y Arqueología - FELAA. Quito, 17-23 de julio del 2011). Quito: Ediciones Abya-Yala/UPS.

RIVADENEIRA NÚÑEZ, Guadalupe

2012 “A propósito de la transversalización de género en un proyecto de desarrollo en la provincia de Santa Elena”, en: Eloy Alfaro R., Katterine Enríquez y Yolanda Flores (comp.). Naturaleza y Cultura en América Latina. Escenarios para un modelo de desarrollo no civilizatorio. (Memorias del XVIII Foro de Estudiantes Latinoamericanos de Antropología y Arqueología - FELAA. Quito, 17-23 de julio del 2011). Quito: Ediciones Abya-Yala/UPS.

RIVADENEIRA SILVA, Marcos

2011 "Proyecto de rescate de Archivos de la provincia de Chimborazo", en: Katterine Enríquez (compilación y edición). La arqueología y la antropología en Ecuador. Escenarios, retos y perspectivas. Quito: Ediciones Abya-Yala/ UPS. 
SANCHEZ-PARGA, José

2011 "El antropólogo entre el oficio y la ciencia”, en: Katterine Enríquez (compilación y edición). La arqueología y la antropología en Ecuador. Escenarios, retos y perspectivas. Quito: Ediciones Abya-Yala/UPS.

SOTOMAYOR, Natalia

2012 "La perspectiva de género en la Antropología”, en: Eloy Alfaro R., Katterine Enríquez y Yolanda Flores (comp.). Naturaleza y Cultura en América Latina. Escenarios para un modelo de desarrollo no civilizatorio. (Memorias del XVIII Foro de Estudiantes Latinoamericanos de Antropología y Arqueología - FELAA. Quito, 17-23 de julio del 2011). Quito: Ediciones Abya-Yala/ UPS.

VANEGAS RODRÍGUEZ, Laura y Luis G. Plazas

2012 "Amazonía: la disputa entre los espacios ancestrales y los intereses imperialistas”, en: Eloy Alfaro R., Katterine Enríquez y Yolanda Flores (comp.). Naturaleza y Cultura en América Latina. Escenarios para un modelo de desarrollo no civilizatorio. (Memorias del XVIII Foro de Estudiantes Latinoamericanos de Antropología y Arqueología - FELAA. Quito, 17-23 de julio del 2011). Quito: Ediciones Abya-Yala/UPS.

VITERI, María Amelia

2011 "Las diversidades y la antropología”, en: Katterine Enríquez (compilación y edición). La arqueología y la antropología en Ecuador. Escenarios, retos y perspectivas. Quito: Ediciones Abya-Yala/UPS.

VOGLIANO, María Soledad y María Fernanda Vallejo

2012 “'Insurgir en el ombligo de la bestia'. Re-existencia y descolonialidad en el seno del desarrollo", en: Eloy Alfaro R., Katterine Enríquez y Yolanda Flores (comp.). Naturaleza y Cultura en América Latina. Escenarios para un modelo de desarrollo no civilizatorio. (Memorias del XVIII Foro de Estudiantes Latinoamericanos de Antropología y Arqueología - FELAA. Quito, 17-23 de julio del 2011). Quito: Ediciones Abya-Yala/UPS. 